\title{
EXISTENCE OF POSITIVE SOLUTIONS FOR A SECOND ORDER \\ PERIODIC BOUNDARY VALUE PROBLEM WITH IMPULSIVE EFFECTS
}

\author{
Jiafa Xu — Zhongli Wei — Youzheng Ding
}

\begin{abstract}
In this paper, we are mainly concerned with the existence and multiplicity of positive solutions for the following second order periodic boundary value problem involving impulsive effects
\end{abstract}

$$
\begin{cases}-u^{\prime \prime}+\rho^{2} u=f(t, u), & \\ -\left.\Delta u^{\prime}\right|_{t=t_{k}}=I_{k}\left(u\left(t_{k}\right)\right), & k=1, \ldots, m, \\ u(0)-u(2 \pi)=0, \quad u^{\prime}(0)-u^{\prime}(2 \pi)=0 . & \end{cases}
$$

Here $J^{\prime}=J \backslash\left\{t_{1}, \ldots, t_{m}\right\}, f \in C\left(J \times \mathbb{R}^{+}, \mathbb{R}^{+}\right), I_{k} \in C\left(\mathbb{R}^{+}, \mathbb{R}^{+}\right)$, where $\mathbb{R}^{+}=[0, \infty), J=[0,2 \pi]$. The proof of our main results relies on the fixed point theorem on cones. The paper extends some previous results and reports some new results about impulsive differential equations.

\section{Introduction}

In this paper, we investigate the existence and multiplicity of positive solutions for the following second order periodic boundary value problem involving

2010 Mathematics Subject Classification. 34B15, 34B18, 34B37, 45M20, 47H10.

Key words and phrases. Periodic boundary value problem, fixed point theorem, positive solution, cone. 
impulsive effects

$$
\begin{cases}-u^{\prime \prime}+\rho^{2} u=f(t, u), & t \in J^{\prime}, \\ -\left.\Delta u^{\prime}\right|_{t=t_{k}}=I_{k}\left(u\left(t_{k}\right)\right), & k=1, \ldots, m, \\ u(0)-u(2 \pi)=0, \quad u^{\prime}(0)-u^{\prime}(2 \pi)=0, & \end{cases}
$$

where $J^{\prime}=J \backslash\left\{t_{1}, \ldots, t_{m}\right\}, J=[0,2 \pi], \rho>0, f \in C\left(J \times \mathbb{R}^{+}, \mathbb{R}^{+}\right), I_{k} \in$ $C\left(\mathbb{R}^{+}, \mathbb{R}^{+}\right), t_{k}(k=1, \ldots, m)$ are fixed points with $0<t_{1}<\ldots<t_{m}<2 \pi$, $\left.\Delta u^{\prime}\right|_{t=t_{k}}=u^{\prime}\left(t_{k}^{+}\right)-u^{\prime}\left(t_{k}^{-}\right)$, where $u^{\prime}\left(t_{k}^{+}\right)$and $u^{\prime}\left(t_{k}^{-}\right)$denote the right and left limit of $u^{\prime}(t)$ at $t=t_{k}$, respectively.

As one kind of instantaneous mutation phenomena, the impulsive phenomenon exists widespread in practical problems in areas of the modern technology, and its mathematical model is always due to the impulsive differential system. Therefore, the impulsive differential equation, developed in recent years, becomes an important branch of the differential equations. This explains the reason that the last two decades have witnessed an overgrowing interest in the research of such problems, with many papers in this direction published.

When $I_{k} \equiv 0$ for $k=1, \ldots, m$, problem (1.1) reduces to the periodic boundary value problem of ordinary differential equation, which has been studied using various theorems and methods of nonlinear functional analysis, see [1], [3], [4], [7], [8], [10], [11], [16], [17], [21]. In [21], Zhang and Wang studied the following periodic boundary value problem

$$
\left\{\begin{array}{l}
-u^{\prime \prime}+\rho^{2} u=f(t, u), \\
u(0)-u(2 \pi)=0, \quad u^{\prime}(0)-u^{\prime}(2 \pi)=0,
\end{array} \quad t \in J,\right.
$$

and established the existence and multiplicity of positive solutions to the problem (1.2) under the condition that $f:[0,2 \pi] \times \mathbb{R}^{+} \rightarrow \mathbb{R}^{+}$is a Carathéodory function.

In recent years, periodic boundary value problems of nonlinear impulsive differential equations have received considerable attention and much literature has been published about the existence of solutions of periodic boundary problems for impulsive differential equations, see, for example, [2], [6], [12]-[15], [18]-[20] In [19], Zhou, Jiang and Tian considered the problem

$$
\begin{cases}-u^{\prime \prime}+\rho^{2} u=f(t, u), & t \in J^{\prime}, \\ \left.\Delta u\right|_{t=t_{k}}=I_{k}\left(u\left(t_{k}\right)\right), \quad-\left.\Delta u^{\prime}\right|_{t=t_{k}}=J_{k}\left(u\left(t_{k}\right)\right), & k=1, \ldots, m, \\ u(0)-u(2 \pi)=0, \quad u^{\prime}(0)-u^{\prime}(2 \pi)=0 . & \end{cases}
$$

The authors adopted a fixed point theorem on a cone to obtain some conditions on $f, I_{k}$ and $J_{k}$, which guarantee the existence of solutions for (1.3).

Motivated by the works mentioned above, in this paper, we study the existence and multiplicity of positive solutions for (1.1). Nevertheless, our methodology and results in this paper are different from those in the papers cited above. We first note the problem (1.1) as a perturbation of the problem (1.2), so we 
can construct an integral operator for the corresponding linear periodic boundary value problem without impulsive effects and find out its first eigenvalue and eigenfunction. Then we establish a special cone associated with the Green function of (1.1). Finally, by employing fixed point index theory, combined with a priori estimates of positive solutions, the existence and multiplicity of positive solutions of (1.1) are obtained. It is of interest to note that $f, I_{k}$ in (1.1) may grow superlinearly and sublinearly. These mean that our methodology and main results here are entirely different from those in [19], [21].

This paper is organized as follows. Section 2 contains some preliminary results. Section 3 is devoted to the existence and multiplicity of positive solutions for (1.1).

\section{Preliminaries}

Let $J^{\prime}=J \backslash\left\{t_{1}, \ldots, t_{m}\right\}$,

$\mathrm{PC}(J, \mathbb{R}):=\{x: J \mapsto \mathbb{R}:$

$$
\left.\left.x\right|_{\left(t_{k}, t_{k+1}\right)} \in C\left(t_{k}, t_{k+1}\right), x\left(t_{k}^{-}\right)=x\left(t_{k}\right), \exists x\left(t_{k}^{+}\right), k=1, \ldots, m\right\}
$$

be a Banach space with norm $\|x\|_{\mathrm{PC}}:=\sup _{t \in[0,2 \pi]}|x(t)|$. Let $P:=\{u \in \operatorname{PC}(J, \mathbb{R})$ : $u(t) \geq 0, t \in J\}$, and then $P$ is a cone in $\operatorname{PC}(J, R)$.

Lemma 2.1 (see [19]). Let $f$ and $I_{k}$ be defined in (1.1). Then the problem (1.1) is equivalent to

$$
u(t)=\int_{0}^{2 \pi} G(t, s) f(s, u(s)) d s+\sum_{k=1}^{m} G\left(t, t_{k}\right) I_{k}\left(u\left(t_{k}\right)\right)=:(A u)(t),
$$

where

$$
G(t, s):=\frac{1}{2 \rho\left(e^{2 \rho \pi}-1\right)} \begin{cases}e^{\rho(t-s)}+e^{\rho(2 \pi-t+s)}, & 0 \leq s \leq t \leq 2 \pi, \\ e^{\rho(s-t)}+e^{\rho(2 \pi-s+t)}, & 0 \leq t \leq s \leq 2 \pi .\end{cases}
$$

Note that if $f \in C\left(J \times \mathbb{R}^{+}, \mathbb{R}^{+}\right), I_{k} \in C\left(\mathbb{R}^{+}, \mathbb{R}^{+}\right)$, then $A: P \rightarrow P$ is a completely continuous operator. From (2.1), we easily know that the existence of positive solutions for (1.1) is equivalent to that of positive fixed points of $A$. Define $\omega:=1 / e^{2 \rho \pi}>0$ and $P_{0}:=\{u \in \operatorname{PC}(J, \mathbb{R}): u(t) \geq \omega\|u\|, t \in J\}$. Clearly, $P_{0}$ is also a cone in $\mathrm{PC}(J, \mathbb{R})$.

Lemma 2.2. $A(P) \subset P_{0}$ (in particular $\left.A\left(P_{0}\right) \subset P_{0}\right)$.

Proof. We note that $G$ has the following properties

$$
\begin{aligned}
\frac{1}{\rho\left(e^{2 \rho \pi}-1\right)}<\frac{e^{\rho \pi}}{\rho\left(e^{2 \rho \pi}-1\right)} & =G(\pi, 0) \leq G(t, s) \\
& \leq G(0,2 \pi)=\frac{e^{2 \rho \pi}+1}{2 \rho\left(e^{2 \rho \pi}-1\right)}<\frac{e^{2 \rho \pi}}{\rho\left(e^{2 \rho \pi}-1\right)}
\end{aligned}
$$


By the definition of $A$ and (2.2), we have

$$
\|A u\| \leq \frac{e^{2 \rho \pi}+1}{2 \rho\left(e^{2 \rho \pi}-1\right)} \int_{0}^{2 \pi} f(s, u(s)) d s+\frac{e^{2 \rho \pi}}{\rho\left(e^{2 \rho \pi}-1\right)} \sum_{k=1}^{m} I_{k}\left(u\left(t_{k}\right)\right) .
$$

On the other hand, by (2.2) again, we find,

$$
\begin{aligned}
(A u)(t) \geq & \frac{e^{\rho \pi}}{\rho\left(e^{2 \rho \pi}-1\right)} \int_{0}^{2 \pi} f(s, u(s)) d s+\frac{1}{\rho\left(e^{2 \rho \pi}-1\right)} \sum_{k=1}^{m} I_{k}\left(u\left(t_{k}\right)\right) \\
= & \frac{2 e^{\rho \pi}}{e^{2 \rho \pi}+1} \cdot \frac{e^{2 \rho \pi}+1}{2 \rho\left(e^{2 \rho \pi}-1\right)} \int_{0}^{2 \pi} f(s, u(s)) d s \\
& +\frac{1}{e^{2 \rho \pi}} \cdot \frac{e^{2 \rho \pi}}{\rho\left(e^{2 \rho \pi}-1\right)} \sum_{k=1}^{m} I_{k}\left(u\left(t_{k}\right)\right) \\
\geq & \frac{1}{e^{2 \rho \pi}}\left[\frac{e^{2 \rho \pi}+1}{2 \rho\left(e^{2 \rho \pi}-1\right)} \int_{0}^{2 \pi} f(s, u(s)) d s+\frac{e^{2 \rho \pi}}{\rho\left(e^{2 \rho \pi}-1\right)} \sum_{k=1}^{m} I_{k}\left(u\left(t_{k}\right)\right)\right] .
\end{aligned}
$$

As a result, $(A u)(t) \geq \omega\|A u\|$, for all $t \in J$ as claimed.

REMARK 2.3. The completely continuous operator $A$, defined by (2.1), satisfies $A(P) \subset P_{0}$ and in particular $A\left(P_{0}\right) \subset P_{0}$. Hence our proof in this paper will be carried out in $P_{0}$ rather than in $P$.

Denote a linear operator by

$$
(L u)(t):=\int_{0}^{2 \pi} G(t, s) u(s) d s, \quad t \in J,
$$

then it is easy to check that $L: C(J) \rightarrow C(J)$ is a completely continuous and positive operator. From now on, let $r(L)$ denote the spectral radii of $L$. By Gelfand's theorem, we have easily the following result.

LEMma 2.4. L's spectral radii $r(L)>0$.

Proof. By the definition of $L$, we have

$$
\|L\|=\max _{t \in J} \int_{0}^{2 \pi} G(t, s) d s \geq \max _{t \in J} \int_{0}^{2 \pi} G(\pi, 0) d s=2 \pi G(\pi, 0)>0 .
$$

Similarly,

$$
\begin{aligned}
\left\|L^{2}\right\| & =\max _{t \in J} \int_{0}^{2 \pi} \int_{0}^{2 \pi} G(t, s) G(s, \tau) d \tau d s \\
& \geq \max _{t \in J} \int_{0}^{2 \pi} \int_{0}^{2 \pi} G(\pi, 0) G(\pi, 0) d \tau d s=[2 \pi G(\pi, 0)]^{2} .
\end{aligned}
$$

Inductively, we have $\left\|L^{n}\right\| \geq[2 \pi G(\pi, 0)]^{n}, n=1,2, \ldots$ Therefore, Gelfand's Theorem gives

$$
r(L)=\inf _{n \geq 1} \sqrt[n]{\left\|L^{n}\right\|} \geq 2 \pi G(\pi, 0)>0
$$


By Lemma 2.4 and the Krein-Rutman Theorem [9], there exist two functions $\varphi \in P \backslash\{0\}$ and $\psi \in P \backslash\{0\}$ with $\psi(x) \geq 0$ for which

$$
\begin{aligned}
\int_{0}^{2 \pi} G(t, s) \varphi(s) d s & =r(L) \varphi(t) \\
\int_{0}^{2 \pi} G(t, s) \psi(t) d t & =r(L) \psi(s), \\
\int_{0}^{2 \pi} \psi(t) d t & =1 .
\end{aligned}
$$

We denote $B_{\rho}:=\{u \in E:\|u\|<\rho\}$ for $\rho>0$ in the sequel.

Lemma 2.5 (see [5]). If $A: \bar{B}_{\rho} \cap P \rightarrow P$ is a completely continuous operator. If there exists $u_{0} \in P \backslash\{0\}$ such that $u-A u \neq \lambda u_{0}$, for all $\lambda \geq 0, u \in \partial B_{\rho} \cap P$, then $i\left(A, B_{\rho} \cap P, P\right)=0$.

Lemma 2.6 (see [5]). If $0 \in B_{\rho}$ and $A: \bar{B}_{\rho} \cap P \rightarrow P$ is a completely continuous operator. If $u \neq \lambda A u$, for all $u \in \partial B_{\rho} \cap P, 0 \leq \lambda \leq 1$, then $i\left(A, B_{\rho} \cap P, P\right)=1$.

\section{Main results}

Let $\lambda_{1}:=1 / r(L)$. We now list our hypotheses.

(H1) There exist $c>0$ and $a_{1} \geq 0, a_{2} \geq 0$ satisfying $a_{1}+\omega a_{2} \sum_{k=1}^{m} \psi\left(t_{k}\right)>\lambda_{1}$, such that

$$
f(t, u) \geq a_{1} u-c, \quad I_{k}(u) \geq a_{2} u-c, \quad \text { for all } t \in[0,2 \pi], u \geq 0 .
$$

(H2) There exist $r>0$ and $b_{1} \geq 0, b_{2} \geq 0$ satisfying

$$
b_{1}^{2}+b_{2}^{2} \neq 0, \quad b_{1}+\omega^{-1} b_{2} \sum_{k=1}^{m} \psi\left(t_{k}\right)<\lambda_{1},
$$

such that

$$
f(t, u) \leq b_{1} u, \quad I_{k}(u) \leq b_{2} u, \quad \text { for all } t \in[0,2 \pi], 0<u<r .
$$

(H3) There exist $r>0$ and $a_{3} \geq 0, a_{4} \geq 0$ satisfying $a_{3}+\omega a_{4} \sum_{k=1}^{m} \psi\left(t_{k}\right)>\lambda_{1}$, such that

$$
f(t, u) \geq a_{3} u, \quad I_{k}(u) \geq a_{4} u, \quad \text { for all } t \in[0,2 \pi], 0<u<r .
$$

(H4) There exist $c>0$ and $b_{3} \geq 0, b_{4} \geq 0$ satisfying

$$
b_{3}^{2}+b_{4}^{2} \neq 0, \quad b_{3}+\omega^{-1} b_{4} \sum_{k=1}^{m} \psi\left(t_{k}\right)<\lambda_{1},
$$

such that

$$
f(t, u) \leq b_{3} u+c, \quad I_{k}(u) \leq b_{4} u+c, \quad \text { for all } t \in[0,2 \pi], u \geq 0 .
$$


(H5) There is a $\delta>0$ such that $0 \leq u \leq \delta$ and $0 \leq t \leq 2 \pi$ implies

$$
f(t, u) \leq \eta \delta, \quad I_{k}(u) \leq \eta_{k} \delta
$$

where $\eta, \eta_{k} \geq 0$ satisfy

$$
\eta+\sum_{k=1}^{m} \eta_{k}>0, \quad 2 \pi \eta+\sum_{k=1}^{m} \eta_{k}<\frac{\rho\left(e^{2 \rho \pi}-1\right)}{e^{2 \rho \pi}} .
$$

(H6) There is a $\delta>0$ such that $\omega \delta \leq u \leq \delta$ and $0 \leq t \leq 2 \pi$ implies

$$
f(t, u) \geq \xi \delta, \quad I_{k}(u) \geq \xi_{k} \delta
$$

where $\xi, \xi_{k} \geq 0$ satisfy

$$
\xi+\sum_{k=1}^{m} \xi_{k}>0, \quad 2 \pi \xi+\sum_{k=1}^{m} \xi_{k}>\rho\left(e^{2 \rho \pi}-1\right) .
$$

ThEOREM 3.1. Suppose that (H1), (H2) are satisfied. Then (1.1) has at least one positive solution.

Proof. Let $\mathcal{M}_{1}=\{u \in P: u=A u+\lambda \varphi, \lambda \geq 0\}$, where $\varphi \in P$ is determined by (2.3). We shall prove that $\mathcal{M}_{1}$ is bounded. Indeed, if $u \in \mathcal{M}_{1}$, then we have $u \geq A u$ by definition. This leads to

$$
u(t) \geq \int_{0}^{2 \pi} G(t, s) f(s, u(s)) d s+\sum_{k=1}^{m} G\left(t, t_{k}\right) I_{k}\left(u\left(t_{k}\right)\right) .
$$

Multiply by $\psi(t)$ on both sides of the above and integrate over $[0,1]$ and use (2.3) to obtain

$$
\begin{aligned}
& \int_{0}^{2 \pi} u(t) \psi(t) d t \\
& \quad \geq \int_{0}^{2 \pi} \psi(t)\left[\int_{0}^{2 \pi} G(t, s) f(s, u(s)) d s+\sum_{k=1}^{m} G\left(t, t_{k}\right) I_{k}\left(u\left(t_{k}\right)\right)\right] d t \\
& \quad=\lambda_{1}^{-1} \int_{0}^{2 \pi} f(t, u(t)) \psi(t) d t+\lambda_{1}^{-1} \sum_{k=1}^{m} \psi\left(t_{k}\right) I_{k}\left(u\left(t_{k}\right)\right) .
\end{aligned}
$$

Consequently,

$$
\lambda_{1} \int_{0}^{2 \pi} u(t) \psi(t) d t \geq \int_{0}^{2 \pi} f(t, u(t)) \psi(t) d t+\sum_{k=1}^{m} \psi\left(t_{k}\right) I_{k}\left(u\left(t_{k}\right)\right) .
$$

Combining this and (3.1), we find

$$
\lambda_{1} \int_{0}^{2 \pi} u(t) \psi(t) d t \geq \int_{0}^{2 \pi} \psi(t)\left(a_{1} u(t)-c\right) d t+\sum_{k=1}^{m} \psi\left(t_{k}\right)\left(a_{2} u\left(t_{k}\right)-c\right) .
$$


From (3.8) we obtain

$$
\left(\lambda_{1}-a_{1}\right) \int_{0}^{2 \pi} u(t) \psi(t) d t+c_{1} \geq a_{2} \sum_{k=1}^{m} u\left(t_{k}\right) \psi\left(t_{k}\right),
$$

where $c_{1}=c\left(1+\sum_{k=1}^{m} \psi\left(t_{k}\right)\right)$. By Lemma 2.2, we have

$$
\left(\lambda_{1}-a_{1}\right)\|u\|+c_{1} \geq \omega a_{2}\|u\| \sum_{k=1}^{m} \psi\left(t_{k}\right) .
$$

Therefore,

$$
\|u\| \leq \frac{c_{1}}{a_{1}+\omega a_{2} \sum_{k=1}^{m} \psi\left(t_{k}\right)-\lambda_{1}}=: \mathcal{N}_{1}, \quad \text { for all } u \in \mathcal{M}_{1},
$$

which establishes the boundedness of $\mathcal{M}_{1}$, as required. Taking $R>\mathcal{N}_{1}$, we obtain

$$
u \neq A u+\lambda \varphi, \quad \text { for all } u \in \partial B_{R} \cap P, \lambda \geq 0 .
$$

Now Lemma 2.5 yields

$$
i\left(A, B_{R} \cap P, P\right)=0 .
$$

Let $\mathcal{M}_{2}:=\left\{u \in \bar{B}_{r} \cap P: u=\lambda A u, \lambda \in[0,1]\right\}$. We shall prove $\mathcal{M}_{2}=\{0\}$. Indeed, if $u \in \mathcal{M}_{2}$, we have

$$
u(t) \leq(A u)(t)=\int_{0}^{2 \pi} G(t, s) f(s, u(s)) d s+\sum_{k=1}^{m} G\left(t, t_{k}\right) I_{k}\left(u\left(t_{k}\right)\right),
$$

for all $u \in \bar{B}_{r} \cap P$. Similar to (3.6), multiply by $\psi(t)$ on both sides of the above and integrate over $[0,1]$ and use $(2.3)$ to obtain

$$
\lambda_{1} \int_{0}^{2 \pi} u(t) \psi(t) d t \leq \int_{0}^{2 \pi} \psi(t) f(t, u(t)) d t+\sum_{k=1}^{m} \psi\left(t_{k}\right) I_{k}\left(u\left(t_{k}\right)\right),
$$

for all $u \in \bar{B}_{r} \cap P$. This, together with (3.2), implies

$$
\lambda_{1} \int_{0}^{2 \pi} u(t) \psi(t) d t \leq b_{1} \int_{0}^{2 \pi} u(t) \psi(t) d t+b_{2} \sum_{k=1}^{m} u\left(t_{k}\right) \psi\left(t_{k}\right) .
$$

Lemma 2.2 leads to

$\omega\left(\lambda_{1}-b_{1}\right)\|u\| \leq\left(\lambda_{1}-b_{1}\right) \int_{0}^{2 \pi} u(t) \psi(t) d t \leq b_{2} \sum_{k=1}^{m} u\left(t_{k}\right) \psi\left(t_{k}\right) \leq b_{2}\|u\| \sum_{k=1}^{m} \psi\left(t_{k}\right)$,

which contradicts the condition $b_{1}+\omega^{-1} b_{2} \sum_{k=1}^{m} \psi\left(t_{k}\right)<\lambda_{1}$. This implies $\mathcal{M}_{2}=$ $\{0\}$ and thus $u \neq \lambda A u$ for all $u \in \partial B_{r} \cap P$ and $\lambda \in[0,1]$. Now Lemma 2.6 yields

$$
i\left(A, B_{r} \cap P, P\right)=1 \text {. }
$$


Combining this with (3.11) gives $i\left(A,\left(B_{R} \backslash \bar{B}_{r}\right) \cap P, P\right)=0-1=-1$. Hence the operator $A$ has at least one fixed point on $\left(B_{R} \backslash \bar{B}_{r}\right) \cap P$ and therefore (1.1) has at least one positive solution.

THEOREM 3.2. Suppose that (H3), (H4) are satisfied. Then (1.1) has at least one positive solution.

Proof. Let $\mathcal{M}_{3}:=\left\{u \in \bar{B}_{r} \cap P: u=A u+\lambda \varphi, \lambda \geq 0\right\}$. We claim $\mathcal{M}_{3} \subset\{0\}$. Indeed, if $u \in \mathcal{M}_{3}$, then we have $u \geq A u$ by definition. By (3.3) and (3.7), we have

$$
\lambda_{1} \int_{0}^{2 \pi} u(t) \psi(t) d t \geq a_{3} \int_{0}^{2 \pi} u(t) \psi(t) d t+a_{4} \sum_{k=1}^{m} u\left(t_{k}\right) \psi\left(t_{k}\right) .
$$

Lemma 2.2 and (3.15) yield

$\left(\lambda_{1}-a_{3}\right)\|u\| \geq\left(\lambda_{1}-a_{3}\right) \int_{0}^{2 \pi} u(t) \psi(t) d t \geq a_{4} \sum_{k=1}^{m} u\left(t_{k}\right) \psi\left(t_{k}\right) \geq \omega a_{4}\|u\| \sum_{k=1}^{m} \psi\left(t_{k}\right)$,

which contradicts the property $a_{3}+\omega a_{4} \sum_{k=1}^{m} \psi\left(t_{k}\right)>\lambda_{1}$. This implies $\|u\| \equiv 0$, for all $u \in \mathcal{M}_{3}$. Hence $\mathcal{M}_{3} \subset\{0\}$, as claimed. As a result, we have $u-A u \neq \lambda \psi$ for all $u \in \partial B_{r} \cap P$ and $\lambda \geq 0$. Now Lemma 2.5 gives

$$
i\left(A, B_{r} \cap P, P\right)=0 .
$$

Let $\mathcal{M}_{4}:=\{u \in P: u=\lambda A u, \lambda \in[0,1]\}$. We assert $\mathcal{M}_{4}$ is bounded. Indeed, if $u \in \mathcal{M}_{4}$, then $u \leq A u$, which can be written in the form

$$
u(t) \leq \int_{0}^{2 \pi} G(t, s) f(s, u(s)) d s+\sum_{k=1}^{m} G\left(t, t_{k}\right) I_{k}\left(u\left(t_{k}\right)\right) .
$$

By (3.4) and (3.12), we obtain

$$
\lambda_{1} \int_{0}^{2 \pi} u(t) \psi(t) d t \leq \int_{0}^{2 \pi} \psi(t)\left(b_{3} u(t)+c\right) d t+\sum_{k=1}^{m} \psi\left(t_{k}\right)\left(b_{4} u\left(t_{k}\right)+c\right) .
$$

From Lemma 2.2, we get

$$
\begin{aligned}
\omega\left(\lambda_{1}-b_{3}\right)\|u\| & \leq\left(\lambda_{1}-b_{3}\right) \int_{0}^{2 \pi} u(t) \psi(t) d t \\
& \leq b_{4} \sum_{k=1}^{m} u\left(t_{k}\right) \psi\left(t_{k}\right)+c_{1} \leq b_{4}\|u\| \sum_{k=1}^{m} \psi\left(t_{k}\right)+c_{1},
\end{aligned}
$$

where $c_{1}=c\left(1+\sum_{k=1}^{m} \psi\left(t_{k}\right)\right)$. Consequently, we see

$$
\|u\| \leq \frac{\omega^{-1} c_{1}}{\lambda_{1}-\left(b_{3}+\omega^{-1} b_{4} \sum_{k=1}^{m} \psi\left(t_{k}\right)\right)}=: \mathcal{N}_{2} .
$$


Now the boundedness of $\mathcal{M}_{4}$, as asserted. Taking $R>\mathcal{N}_{2}$, we have $u \neq \lambda A u$ for all $u \in \partial B_{R} \cap P$ and $\lambda \in[0,1]$. Now Lemma 2.6 yields

$$
i\left(A, B_{R} \cap P, P\right)=1 .
$$

Combining this with (3.16) gives $i\left(A,\left(B_{R} \backslash \bar{B}_{r}\right) \cap P, P\right)=1-0=1$. Hence the operator $A$ has at least one fixed point on $\left(B_{R} \backslash \bar{B}_{r}\right) \cap P$ and therefore (1.1) has at least one positive solution.

TheOREm 3.3. Suppose that (H1), (H3) and (H5) are satisfied. Then (1.1) has at least two positive solutions.

Proof. By (H5) and (2.2), we have

$$
\begin{aligned}
\|A u(t)\| & \leq \int_{0}^{2 \pi} \frac{e^{2 \rho \pi}}{\rho\left(e^{2 \rho \pi}-1\right)} \eta \delta d s+\sum_{k=1}^{m} \frac{e^{2 \rho \pi}}{\rho\left(e^{2 \rho \pi}-1\right)} \eta_{k} \delta \\
& =\frac{e^{2 \rho \pi} \delta}{\rho\left(e^{2 \rho \pi}-1\right)}\left[2 \pi \eta+\sum_{k=1}^{m} \eta_{k}\right]<\delta .
\end{aligned}
$$

Consequently, $\|A u\|<\|u\|$, for all $u \in \partial B_{\delta} \cap P$, and thus $u \neq \lambda A u$ for all $u \in \partial B_{\delta} \cap P$ and $\lambda \in[0,1]$. Now Lemma 2.6 yields

$$
i\left(A, B_{\delta} \cap P, P\right)=1 .
$$

On the other hand, in view of (H1) and (H3), we may choose $R>\delta$ and $r \in$ $(0, \delta)$ such that (3.11) and (3.16) hold (see the proofs of Theorems 3.1 and 3.2). Together with (3.11), (3.16) and (3.18), we have

$$
i\left(A,\left(B_{R} \backslash \bar{B}_{\delta}\right) \cap P, P\right)=0-1=-1, \quad i\left(A,\left(B_{\delta} \backslash \bar{B}_{r}\right) \cap P, P\right)=1-0=1 .
$$

Therefore, $A$ has at least two fixed points, one on $\left(B_{R} \backslash \bar{B}_{\delta}\right) \cap P$ and the other on $\left(B_{\delta} \backslash \bar{B}_{r}\right) \cap P$. Equivalently, (1.1) has at least two positive solutions.

TheOrem 3.4. Suppose that (H2), (H4) and (H6) are satisfied. Then (1.1) has at least two positive solutions.

Proof. By Lemma 2.2, red for all $u \in \partial B_{\delta} \cap P$ and $t \in[0,2 \pi]$, we obtain $\omega \delta \leq u(t) \leq \delta$. Therefore, from (H6) and (2.2), we have

$$
\begin{aligned}
\|A u(t)\| & \geq \int_{0}^{2 \pi} \frac{1}{\rho\left(e^{2 \rho \pi}-1\right)} \xi \delta d s+\sum_{k=1}^{m} \frac{1}{\rho\left(e^{2 \rho \pi}-1\right)} \xi_{k} \delta \\
& =\frac{\delta}{\rho\left(e^{2 \rho \pi}-1\right)}\left[2 \pi \xi+\sum_{k=1}^{m} \xi_{k}\right]>\delta .
\end{aligned}
$$

Consequently, we find $u-A u \neq \lambda \varphi$, for all $u \in \partial B_{\delta} \cap P$ and $\lambda \geq 0$. We obtain by Lemma 2.5

$$
i\left(A, B_{\delta} \cap P, P\right)=0 .
$$


On the other hand, in view of (H2) and (H4), we may choose $R>\delta$ and $r \in(0, \delta)$ such that (3.14) and (3.17) hold (see the proofs of Theorem 3.1 and 3.2). We obtain by (3.14), (3.17) and (3.20)

$$
i\left(A,\left(B_{R} \backslash \bar{B}_{\delta}\right) \cap P, P\right)=1-0=1, \quad i\left(A,\left(B_{\delta} \backslash \bar{B}_{r}\right) \cap P, P\right)=0-1=-1 .
$$

Hence $A$ has at least two fixed points, one on $\left(B_{R} \backslash \bar{B}_{\delta}\right) \cap P$ and the other on $\left(B_{\delta} \backslash \bar{B}_{r}\right) \cap P$, and thus (1.1) has at least two positive solutions.

\section{REFERENCES}

[1] J. ChU, X. LIN AND D. JIANG, Multiplicity of positive periodic solutions to second order differential equations, Bull. Austral. Math. Soc. 73 (2006), 175-182.

[2] W. Ding, J. Mi And M. Han, Periodic boundary value problems for the first order impulsive functional differential equations, Appl. Math. Comput. 165 (2005), 443-456.

[3] D. Franco AND J. WeBB, Collisionless orbits of singular and nonsingular dynamical systems, Discrete Contin. Dyn. Syst. 15 (2006), 747-757.

[4] H. GaO, S. Weng, D. JiAng And X. Hou, On second order periodic boundary value problems with upper and lower solutions in the reversed order, Electron. J. Differential Equations 126 (2005), 1-10.

[5] D. Guo and V. Lakshmikantham, Nonlinear Problem in Abstract Cones, Academic Press, New York, 1988.

[6] Z. HE, X. HE, Periodic boundary value problems for first order impulsive intergrodifferential equations of mixed type, J. Math. Anal. Appl. 296 (2004), 8-20.

[7] D. JiAng, M. FAnA AND A. WAN, A monotone method for constructing extremal solutions to second-order periodic boundary value problems, J. Comput. Appl. Math., 136 (2001), 189-197.

[8] D. JiAng, J. Nieto AND W. Zuo, On monotone method for first and second order periodic boundary value problems and periodic solutions of functional differential equations, J. Math. Anal. Appl. 289 (2004), 691-699.

[9] M. Krein, M. Rutman, Linear operators leaving invariant a cone in a Banach space, Uspekhi Mat. Nauk 23 (1948), 3-95.

[10] Y. LI, Positive periodic solutions of first and second order ordinary differential equations, Chin. Ann. Math. Ser. B 25 (2004), 413-420.

[11] X. Lin, X. Li AND D. JIANG, Positive solutions to superlinear semipositone periodic boundary value problems with repulsive weak singular forces, Comput. Math. Appl. 51 (2006), 507-514.

[12] R. Liang And J. Shen, Periodic boundary value problem for the first order impulsive functional differential equations, J. Comput. Appl. Math. 220 (2007), 498-510.

[13] J. Li AND J. SHen, Periodic boundary value problems for second order differential equations with impulses, Nonlinear Stud. 124 (2005), 391-400.

[14] Z. LuO AND J. NiETo, New results for the periodic boundary value problem for impulsive integro-differential equations, Nonlinear Anal. 70 (2009), 2248-2260.

[15] J. Nieto AND R. Rodríguez-López, New comparison results for impulsive integrodifferential equations and applications, J. Math. Anal. Appl. 328 (2007), 1343-1368.

[16] P. TORREs, Non-collision periodic solutions of forced dynamical systems with weak singularities, Discrete Contin. Dyn. Syst. 11 (2004), 693-698. 
[17] P. TORRES, Existence of one-signed periodic solutions of some second-order differential equations via a Krasnosel'skiǔ fixed point theorem, J. Differential Equations 190 (2003), 643-662.

[18] X. YANG AND J. Shen, Periodic boundary value problems for second-order impulsive integro-differential equations, J. Comput. Appl. Math. 209 (2007), 176-186.

[19] Q. Zhou, D. JiAng And Y. Tian, Multiplicity of positive solutions to period boundary value problems for second order impulsive differential equations, Acta Mathematicae Applicatae Sinica, English Series, vol. 26, 2010, pp. 113-124.

[20] X. ZhANG, X. Li, D. JiAng AND K. WANG, Multipilicity positive solutions to periodic problems for first order impulsive differential equations, Comput. Math. Appl. 52 (2006), 953-966.

[21] Z. ZhANG AND J. WANG, On existence and multiplicity of positive solutions to periodic boundary value problems for singular nonlinear second order differential equations, J. Math. Anal. Appl. 281 (2003), 99-107.

Manuscript received September 23, 2011

JiAFA XU

School of Mathematics

Shandong University

Jinan, Shandong, 250100, P.R. CHINA

E-mail address: xujiafa292@sina.com, jiafaxu@sina.cn

Zhongli WeI AND Youzheng Ding

School of Mathematics

Shandong University

Jinan, Shandong, 250100, P.R. CHINA

and

Department of Mathematics

Shandong Jianzhu University

Jinan, Shandong, 250101, P.R. CHINA

E-mail address: jnwzl132@163.com, dingyouzheng@139.com 